\title{
Primer Congreso Interuniversitario de Educación Virtual: Digital World Learning CIEV
}

\section{First Interuniversity Congress of Virtual Education: Digital World Learning CIEV}

\section{Referencia del resumen}

\author{
- Karla Lisseth Valdez Hernández \\ - Universidad Galileo de Guatemala \\ kvaldez@fahusac.edu.gt \\ https://orcid.org/0000-0002-1114-7898
}

Valdez, K. (2020). Primer Congreso Interuniversitario de Educación Virtual: Digital World Learning CIEV 2018. Revista Guatemalteca de Educación Superior, 3(1), 116-126.

DOI: https://doi.org/10.46954/revistages.v111.9

Recibido 18/05/2020.

Revisado 20/05/2020.

Aceptado 22/06/2020.

\section{Resumen}

La educación se ha transformado y modernizado implementando nuevos escenarios de aprendizaje. Cada vez más, son los directivos de las instituciones educativas a nivel superior quienes han realizado esfuerzos para establecer unidades o departamentos de educación virtual en beneficio de la comunidad educativa para fortalecer la práctica docente.

Con la evolución de la tecnología educativa ha sido posible la constante actualización en los profesionales de la educación, logrando establecer competencias y habilidades informacionales y digitales que todo docente debe poseer en pleno siglo XXI; el 
educador en la era digital debe ser capaz de aplicar las TIC, TAC, TEP para mediar el proceso de enseñanza aprendizaje. Por medio de las tecnologías de la información y comunicación (TIC), se posibilita la difusión, intercambio y adquisición de la información; seguidamente evoluciona a las tecnologías del aprendizaje y conocimiento (TAC) como aspecto menos instrumental y más de formación en la adquisición de los saberes (ser y hacer) para culminar con las tecnologías para el empoderamiento y participación (TEP), que hacen referencia en participar con la tecnología. Todo lo anterior, con el fin de comunicar, transformar, solucionar y crear tendencias para mejorar los entornos de aprendizaje en los estudiantes para la autorregulación, autodeterminación y autorrealización personal.

\section{Abstract}

Education has been transformed and modernized by implementing new learning scenarios. Increasingly, it is the directors of educational institutions at the higher level who have made efforts to establish virtual education units or departments for the benefit of the educational community in order to strengthen teaching practice.

With the evolution of educational technology, constant updating has been possible in education professionals, managing to establish informational and digital competences and skills that every teacher must possess in the XXI century; the educator in the digital age must be able to apply ICT, TAC, TEP to mediate the teaching-learning process. Through information and communication technologies (ICT), the dissemination, exchange and acquisition of information is made possible; It then evolves to the technologies of learning and knowledge (TAC) as a less instrumental aspect and more of training in the acquisition of knowledge (being and doing) and culminating in the technologies for empowerment and participation (TEP), which refer in participating with technology, achieving: communicating, transforming, solving and creating trends to improve learning environments in students for self-regulation, self-determination and personal self-realization.
Palabras clave:

educación, digital, congreso.
Keywords:

education, digital, congress, trends 


\section{Introducción}

El Primer Congreso Interuniversitario de Educación Virtual se llevó a cabo en las instalaciones de la Facultad de Humanidades de la Universidad de San Carlos de Guatemala. Surgió como iniciativa académica y científica con el objetivo de fortalecer el proceso de enseñanza aprendizaje mediados por las tecnologías de la información y comunicación; con el propósito de socializar conocimientos, investigaciones y experiencias de buenas prácticas en el ejercicio docente en las modalidades semipresencial, virtual y a distancia. Se abordaron temáticas en la aplicación de las TIC desde la dimensión pedagógica para contribuir y mejorar la práctica docente superior.

Dentro de los ejes temáticos se encuentra la calidad en la educación superior virtual, estrategias en el aula virtual, diseño instruccional, uso de herramientas web 2.0 y MOOC, entre otros. El comité organizador fue integrado por profesionales de la Facultad de Humanidades de la Universidad de San Carlos de Guatemala, Universidad Mariano Gálvez y Universidad Galileo. Se convocó a profesionales del área de gestión del conocimiento, investigadores, profesores, formación empresarial y estudiantes, quienes utilizan la tecnología educativa como un medio para desarrollar las competencias de aprendizaje en los estudiantes y los objetivos de enseñanza establecidos por los profesores de las modalidades educativas semipresencial, virtual y a distancia. El evento contó con dos conferencias magistrales, seis talleres y veinte ponencias.

De las dos conferencias magistrales, en la sesión inaugural, la Dra. Carina Grisolía de la República de Argentina dictó la conferencia titulada: “¿Cómo potenciar el aprendizaje virtual? las salas de e learning”. En la sesión de cierre, la conferencia magistral titulada "La cuarta revolución industrial, una nueva economía, una nueva educación”, fue dictada por el Dr. Rocael Hernández, Director de GES (Galileo Educational System) de Universidad Galileo.

El procedimiento para la aceptación de las ponencias se detalla a continuación: primero se hizo el llamado para el envío de las contribuciones en dos categorías por medio de la página web 
del congreso. Estas eran Full paper - artículos de investigación y experiencias (hasta 6 páginas) y Short paper - artículos cortos de trabajo en progreso (hasta 4 páginas).

Acerca de la información de envío y publicación: los trabajos se prepararon a través de las directrices de Springer LNCS. EI medio de recepción fue mediante la plataforma EasyChair y los artículos fueron publicados en el libro de actas CEUR, que es una plataforma de libre acceso para conferencias y talleres científicos.

Una vez cerrada la fecha para la entrega de artículos, el comité científico procedió a la revisión de la temática, redacción, estilo y estructura del artículo, para luego validar las contribuciones enviadas. En algunos casos, los artículos fueron regresados a los autores con observaciones de dicho comité para la mejora del documento, y fue enviado de nuevo para su aceptación final y publicación.

Las ponencias aceptadas fueron distribuidas cinco disertaciones en cuatro bloques de sesión, con una duración de veinte minutos, para un total de veinte ponencias disertadas. Hubo un espacio para que los participantes intervinieran con preguntas 0 aportes, para lo cual fueron programados quince minutos para resolverlos. El moderador asignado para cada bloque fue el responsable de dirigir el acto académico.

Por último, en cuanto a la ejecución de los seis talleres, fueron distribuidos en dos jornadas de actividad simultánea, dirigidos por dos profesionales de cada universidad involucrada en la organización. Se atendió a los participantes en laboratorios de computación y en salones de clases. Se abordaron temas como el aprendizaje en tiempos de MOOC: Diseñando mi curso virtual en formato MOOC, aplicación del aprendizaje colaborativo en ambientes de aprendizaje virtual, ¿Cómo aprenden las personas en la virtualidad? ¡Pero si es tan fácil!, feedback en el aprendizaje virtual! ¿Se lo digo o no se lo digo?, Gamification como estrategia didáctica en un entorno virtual de aprendizaje (EVA) y Facebook live en el proceso de enseñanza aprendizaje. 


\section{Contenido}

Ideas críticas para el diseño integral de experiencias de aprendizaje complejo en entornos virtuales. Ponente: Mónica Sulecio de Álvarez. "El aprendizaje que vale la pena es aquel que permite a las personas "ser" y "hacer" mejor, que empodera y que les permite ser competentes en su desempeño, tomando decisiones que acarrean impactos positivos para sí mismos y sus entornos". (Sulecio, 2018)

El aprendizaje que se establece a lo largo de la vida es el aprendizaje que se toma como punto de partida para el desempeño integral de un ser humano en sus diferentes contextos en donde se desenvuelve. Es así como es necesario establecer acciones que provean a los estudiantes una visión global de su quehacer profesional y personal cuando les corresponde enfrentarse a diversas situaciones de la vida que requieren la toma de decisiones y que sean capaces de aportar soluciones para el bien común en beneficio de su propio bienestar, seguridad y el de los demás.

Dentro de las cinco ideas críticas para implementar un diseño curricular y así potenciar los beneficios de aprendizaje y autoaprendizaje que ofrece y posibilita las tecnologías, se detalla lo siguiente:

Garantía de la transferencia del aprendizaje, la creencia potenciadora: la transferencia de lo aprendido al ámbito de la vida real (trabajo, familia, vida cotidiana), se logra cuando la situación de aprendizaje es la vida real misma o un escenario muy similar.

Desde que se inicia la planificación didáctica se hace relevante establecer qué es lo que el estudiante aprenderá; saber qué, cómo, cuándo, dónde y por qué es importante el contenido que desarrollará por medio de actividades de aprendizaje. Se puede decir que se está realizando a la vez concientización de su adquisición de conocimiento, habilidades y destrezas para afianzar el objetivo de enseñanza y cumplir con las competencias fijadas y aplica para lo presencial y virtual. 
Reto para activar el aprendizaje, la creencia potenciadora: partimos de los niveles altos de pensamiento para generar interés por aprender. La taxonomía de Benjamín Bloom, recientemente revisada, clasifica el dominio cognitivo en seis categorías: Recordar, Comprender, Aplicar, Analizar, Evaluar y Crear, al motivar el aprendizaje desde esa utilidad y desde el interés genuino por el nuevo conocimiento se garantiza la transferencia.

Al utilizar la taxonomía de Bloom en los niveles superiores, el aprendizaje del estudiante se ve empoderado puesto que es más significativo "hacer" que solo "saber". Por eso, al considerar implementar evidencias de aprendizaje que demuestren el desempeño logrado, motivará cada vez más a que el estudiante se vea retado para hacer lo que se proponga sin dificultad y sea capaz de construir sus propias ideas al aplicar ese nuevo conocimiento generado.

Apoyo para el aprendizaje, la creencia potenciadora: enseñar es ayudar a aprender, y ayudar a aprender es apoyar el desempeño. Debido a que el reto se plantea a un nivel alto, los aprendices necesitan apoyarse en algo para alcanzarlo". Cada vez que se planteen los retos de aprendizaje, deben ser acordes al nivel cognitivo en el que estudiante se encuentra, es decir, que las evidencias por desempeño deben ser de "reto" pero a la vez alcanzables para ellos al momento de desarrollar su actividad.

Marco filosófico del diseño, argumenta la creencia potenciadora "los métodos, técnicas y teorías se combinan para diseñar experiencias de aprendizaje completas". Con alguna frecuencia, en los espacios educativos se observa cierta oposición o tensión entre quienes prefieren métodos que se acercan más a la teoría cognitivista y aquellos que se inclinan por el constructivismo y el constructivismo social".

Es oportuno emplear un marco de diseño bajo un modelo instruccional filosófico como los que detalla la autora, para que fundamente el modelo educativo de la institución en donde recaerá el proceso de enseñanza aprendizaje. Debe estar estructurado para lograr la planificación didáctica deseada en los entornos virtuales de aprendizaje mediante aspectos que 
sean claros, precisos y concisos, y que no exista un desfase en lo que se quiere enseñar y lograr con los estudiantes. Posicionar un modelo de diseño instruccional facilitará el proceso educativo en los ambientes virtuales.

Enfoque sistémico del diseño de experiencias de aprendizaje, la creencia potenciadora es "ayudar a aprender desde el enfoque de aprendizaje basado en el desempeño o el aprendizaje complejo, implica crear un ecosistema de actividades y recursos que lo posibiliten".

Desde que las tecnologías de la información y comunicación se adentraron en el ámbito educativo, resulta posible que una teoría de aprendizaje sea el fundamento para analizar, diseñar, desarrollar, implementar y evaluar entornos que sean utilizados como ecosistemas de aprendizaje para la generación de habilidades, actitudes, conocimiento y destrezas, para que el estudiante reflexione sobre su adquisición de lo que está aprendiendo.

Motivando la actualización docente con el uso de insignias digitales. Ponentes: Angélica Rocha y Cynthia C. Castillo. Ser educador implica como cualquier otra profesión la constante actualización para reafirmar, rectificar y mejorar procesos cognitivos, habilidades y destrezas que son productos obtenidos a través un periodo de formación previa en niveles de pregrado, grado y posgrado del área académica. Para lograrlo, el docente debe tener la disposición de seguir aprendiendo nuevos saberes y de reaprender los procesos mencionados, para evitar que su quehacer educativo sea limitado y estancado.

En el marco de lo anteriormente escrito, las autoras Rocha \& Castillo (2018) expresan que los educadores universitarios deben estar abiertos a responder a los rápidos cambios en la tecnología y su impacto en la educación. Por tal razón exponen en que, en vez de ofrecer una actualización docente bajo un modelo de taller de capacitación dentro de un espacio físico, saturado de horas de trabajo y que en algunos casos se tornan poco efectivas en la puesta en práctica, decidieron responder al uso de las tecnologías de la información y comunicación, mediante la educación virtual. 
Para dicho curso de actualización, implementaron el curso de "Aprende Canvas", se implementó por la necesidad de buscar una estrategia que motivara más el aprendizaje de los docentes y que su vez permitiera llevar un mejor control de las destrezas adquiridas por los docentes en el uso de la plataforma. Fue así como surgió la idea de integrar insignias digitales dentro del curso".

El curso en línea aborda los temas de enfoque basado por competencias profesionales, tutoría virtual y aprende canvas. Se integra al curso las insignias digitales para la promoción e incentivo de un logro alcanzado o de una habilidad, competencia o destreza obtenida que se puede evidenciar y verificar al acceder en línea a través de un entorno virtual de aprendizaje.

En resumen, con este caso de éxito ejecutado en la Universidad del Valle de Guatemala, se pone en manifiesto que la integración de las TIC en el proceso formativo continuo del profesorado, puede ser una experiencia enriquecedora para fomentar el deseo de continuar con el desarrollo de capacitación y seguir potencializando la práctica educativa superior, al acreditar y certificar las habilidades blandas y duras.

La importancia del tutor en los cursos virtuales: experiencia, buenas prácticas y recomendaciones. Ponentes: Flor Sagastume, Miguel Morales, Héctor Amado y Rocael Hernández. Esta contribución de trabajo da a conocer la necesidad de que las instituciones educativas deben contar con profesionales formados para ser tutores virtuales y así mediar el proceso de enseñanza aprendizaje de manera efectiva; en donde consideran que un tutor virtual debe poseer competencias técnicas, tecnológicas, de comunicación asertiva y la capacidad de establecer la construcción del conocimiento al facilitar herramientas y teniendo en cuenta el oportuno seguimiento, monitoreo, retroalimentación del aprendizaje y moderación en la formación de los estudiantes.

Para lo cual, afirman Sagastume \& Hernández (2018) para moderar la comunicación e interacción en línea, apoyar, guiar, motivar la participación de los estudiantes y promover su autonomía, la Dra. 
Gilly Salmon diseñó un modelo de e-Moderación estructurado en 5 etapas durante las cuales se brinda apoyo técnico y moderación a los estudiantes durante los cursos virtuales.

Para ejemplificar las etapas detalladas según Salomón (2014) la etapa 1 - Acceso y motivación, se da la bienvenida al estudiante y se asegura que no tenga inconvenientes con el acceso y uso de la plataforma de estudio. Etapa 2 - Socialización en línea: se crea un ambiente confortable para los estudiantes y se motiva su participación a través del envío y recepción de mensajes, se promueve que los estudiantes establezcan su identidad. Etapa 3 - Intercambio de información: se asignan tareas acerca del contenido del curso y de la web, se utiliza software personalizado (software para videoconferencias, herramientas web 2.0, etc.). Los estudiantes tendrán interacción con contenidos, con el tutor y compañeros del curso. Etapa 4 - Construcción de conocimiento: se promueve que los estudiantes desarrollen ideas a través de la discusión y la colaboración. Se construye el conocimiento a través de grupos con situaciones y experiencias de la vida real. Etapa 5 - Desarrollo: Los estudiantes se vuelven responsables de su propio aprendizaje, se motiva la reflexión y el pensamiento crítico.

En virtud de lo compartido, el rol que juega el tutor virtual en la educación es de ser sumamente responsable, comprometido, organizado, mediador, facilitador, empático, doblemente comunicativo para que las instrucciones sean claras, precisas y concisas. Debe ser también, guía, gestor en la solución de problemáticas educativas en la virtualidad, entre otras características que lo diferencian a de un profesor en la modalidad educativa presencial. Requiere poseer liderazgo y motivación que contribuya a la práctica docente con calidad académica.

\section{Conclusión}

Los resultados obtenidos en el Primer Congreso Interuniversitario fueron enriquecedores con sustento investigativo, puesto que se dio a conocer por vez primera a la sociedad guatemalteca y a la comunidad de educación superior los avances en la implementación de la educación virtual en las instituciones 
públicas y privadas, que han sido visionarias para estar a la vanguardia y modernizar la academia, convirtiéndola en inclusiva mediante el uso de las tecnologías de la información y comunicación, democratizando la educación para que sea accesible y continuar con el desarrollo social de las comunidades en un país.

\section{Referencias}

Rocha, A., \& Castillo, C. (2018). Motivando la atualización docente con el uso de insignias digitales. I Congreso Interuniversitario de Educación Virtual: Digital World Learning (págs. 8-13). Guatemala: https://www.galileo. edu/page/wp-content/uploads/2019/02/index.html.

Sagastume, F., Morales, M., Amado, H., \& Hernández, R. (2018). La importancia del tutor en los cursos virtuales: experiencia, buenas prácticas y recomendaciones. I Congreso Interuniversitario de Educación Virtual: Digital World Learning (págs. 91-97). Guatemala: https://www. galileo.edu/page/wp-content/uploads/2019/02/index. html.

Salmon, G. (2004). e - Moderating: The key to teaching \& learning online. (págs. 4-100). London:

Faculty of Business and Law (FBL). https://doi. org/10.4324/9780203465424

Sulecio, M. (2018). Ideas críticas para el diseño integral de experiencias de aprendizaje complejo en entornos virtuales. I Congreso Interuniversitario de Educación Virtual: Digital World Learning (págs. 100 - 117). Guatemala: https://www.galileo.edu/page/wp-content/ uploads/2019/02/index.html. 


\section{Sobre la autora}

Karla Valdez es profesional egresada de la Escuela de Estudios de Posgrado de la Facultad de Humanidades de la USAC, como magister en docencia universitaria. Experiencia en investigación generada a través de la tesis titulada: "Los diseños instruccionales para la implementación de cursos en entornos virtuales de aprendizaje y su evidencia de aprendizaje en la educación superior" en Universidad Galileo de Guatemala.

\section{Copyright (c) Karla Lisseth Valdez Hernández}

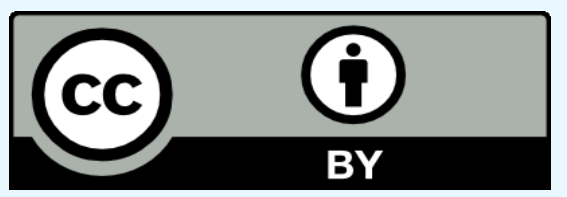

Este texto está protegido por una licencia CreativeCommons 4.0.

Usted es libre para compartir, copiar y redistribuir el material en cualquier medio o formato y adaptar el documento, remezclar, transformar y crear a partir del material para cualquier propósito, incluso comercialmente, siempre que cumpla la condición de atribución: usted debe reconocer el crédito de una obra de manera adecuada, proporcionar un enlace a la licencia, e indicar si se han realizado cambios. Puede hacerlo en cualquier forma razonable, pero no de forma tal que sugiera que tiene el apoyo del licenciante o lo recibe por el uso que hace. 